\title{
REHABILITATION OF PATIENTS WITH APICAL PERIODONTAL CYSTS OF JAWS
}

\section{Yuri Efimov ${ }^{1} \mathbb{D}^{\mathrm{D}}$, Dmitry Stomatov ${ }^{2}$ (D), Evgeniya Efimova ${ }^{1}$, Alexander Stomatov ${ }^{2}$ (D), Ivan Khimich ${ }^{1}$ (D), Pavel Kireev ${ }^{1 凶}$}

\author{
${ }^{1}$ Volgograd State Medical University, Volgograd \\ ${ }_{2}^{2}$ Medical Institute of Penza State University, Penza, Russia
}

kireev-27@yandex.ru
Received 8 June 2020; Received in revised form $30 \mathrm{July} 2020$; Accepted 7 August 2020

\section{The objective of the study}

is to evaluate the efficacy of bone grafting material «bioOst» in filling of infected bone defects.

\section{MATERIALS AND METHODS}

Surgical treatment of 63 patients with apical periodontal cysts of the jaws was performed. In the experimental group ( $31-49,2 \%$ of patients) a bone defect was filled with osteoplastic material «OsteoBiol GenOs» by Tecnoss (Italy). The material is bone granules with collagen / heterologous cortical-spongious mixed (pigs/horse) in the size of 250-1000 microns.

In the control group $(32-50,8 \%$ of patients) osteoplastic matrix «bioOST-XENOGRAFT Collagen» - bone chips with underlying subtotal osteoinductive demineralization, made by $\ll \mathrm{OOO}$ Cardioplant» (Russia) was employed.

Cystectomy was performed using Parch II technique. Three, six and nine months after surgery a case follow-up was done. Activity in regeneration processes was assessed by taking a dental panoramic radiogram using Adobe Photoshop 7,0 software and calculating the coefficient of the bone mineral density in the parietal and middle area of the osseous defect.

The probability of obtained data was calculated by using the Student t-test (reliable data were at $\mathrm{p}<0,05)$.

The conducted survey was approved by the local ethics committee of the Volgograd State Medical University (protocol № 2098-2019 dd 1.03.2019).

\section{RESULTS}

It has been estimated that before surgery in both clinical groups tissue density indexes in the parietal and middle area of the osseous defect showed no statistically significant differences $(p>0,05)$ and were 2 times lower than intact bone indexes.

Three months after surgery in both clinical groups the coefficient of the bone mineral density in the parietal area of the osseous defect equaled to $p>0,05$. At the same time, in the control group difference of the coefficient of the bone mineral density in the parietal area $(128,34 \pm 1,43$ y.e) and middle area of the osseous defect $(124,67 \pm 1,47$ y.e $)$ was statistically invalid, but in the experimental group it was pronounced (125,41 $\pm 1,37$ y.e and $120,27 \pm 1,34$ y.e; $\mathrm{p}<0,05)$. The coefficient of the bone mineral density in middle area of the osseous defect in patients of the ess [2]. 
control group was significantly greater $(p<0,05)$ than in patients of the experimental group.

Six months after surgery there were no statistically significant differences $(p>0,05)$ between the groups in the coefficient of the bone mineral density over the entire area, but they were lower in the intact bone $(\mathrm{p}<0,001)$. Nine months after surgery the coefficient of the bone mineral density over the entire area equaled to the values in the intact bone $(p>0,05)$.

So, the results of the survey showed that processes of reparative osteogenesis started in the parietal area of the osseous defect in both groups, i.e. at the site of a bone grafting material adjacent to the wall of the defect and were directed to the apex. It is termed as creeping substitution meaning the initial resorption of the graft followed by secondary ingrowth of a new bone from the osseous defect. Synchronism of these processes must be emphasized which occurs simultaneously being linked with the degree of demineralization of the implant induced into the osseous defect. In series of studies it is noted that resorption of bone grafting materials with total demineralization occurs to a much greater extent than osteogenesis. Partially demineralized bone implants (interfacial, subtotal demineralization) possess higher osteoinductive activity supporting simultaneous occurrence of biodegradation and osteogenesis processes.

In the survey under study, in both groups simultaneous occurrence of biodegradation and reparative osteogenesis processes was established. At the same time, statistical analysis of the obtained data confirmed a pronounced activity of these processes in using indigenous bone grafting material «bioOst» with interfacial demineralization during the first three months after surgery. To the sixth month of monitoring the rate of reparative osteogenesis and resorption of implant material became even. To the ninth month of a post - operative period complete restoration of the osseous defect was observed.

\section{CONCLUSION}

Indigenous bone grafting material «bioOst» with interfacial demineralization in filling of infected bone defects possesses both osteoinductive and osteoconductive properties contributing to simultaneous occurrence of biodegradation and reparative osteogenesis processes. The advantage of this material is successful integration during the first three months after surgery. That is very essential in prognosis of clinical complications. This evidence based study underlines the necessity to conduct further investigations to reveal the efficacy of the material in filling of infrabony defects of different original.

\section{REFERENCES}

1. Efimov Yu. V., Stomatov D. V., Efimova E. Yu., Stomatov A. V., Dolgova I. V. Treatment of patients with unilateral oblique fracture of the mandible. Medical News of North Caucasus. 2019;14(1.1): 94-97. https://doi.org/10.14300/mnnc.2019.14059

2. Stomatov D.V., Efimov Yu.V., Stomatov A.V., Nesterov A.V., POPOUdin A.A., BODACHEVSKY D.V., KurJaev I.I. The application of the dental implant placement technique with simulated collagen subtotal demineralized cube in Single-step dental implantation of placing. Stomatology for Allю International Dental Review. 2019;2(87):54-57 https://doi. org/10.35556/idr-2019-2(87)54-57

3. Bonavolontà P., Dell'Aversana Orabona G., Friscia M., Sani L. ET AL. Surgical management of large odontogenic cysts of the mandible. The Journal Craniofacacial Surgery. 2019;30(7):658-661. https:// doi.org/10.1097/SCS.0000000000005725.

4. Hahn H.M., Lee Y.J., Park D.H. Huge radicular cyst of the maxilla treated with complete resection and immediate reconstruction by rib bone graft. Journal of Oral and Maxillofacial Surgery. 2019;18:378-381. https://doi.org/10.1007/s12663-018-1125-0

5. Tian F.C., Bergeron B.E., Kalathingal S., MORRIS M. ET. AL. Management of large radicular lesions using decompression: a case series and review of the literature. The Journal of Endodontics. 2019;45(5):651-659. https://doi.org/10.1016/j. joen.2018.12.014.

6. Tiwari S., REDDY S. Management of radicular cyst of primary molar: Case report and literature review. Journal of Advances in Medicine and Medical Research. 2017;22(2):1-6. https://doi.org/10.9734/ BJMMR/2017/33671 